\title{
Computer Simulation of Inverse Problems of Crystal Growth and Photoconductivity of Graded Band Gap Semiconductors
}

\author{
S. V. Kletskii \\ Institute for Semiconductor Physics, Academy of Sciences of Ukraine \\ Prospekt Nauki, 45, SU-252028 Kiev, UKRAINE
}

\begin{abstract}
Some problems of semiconductor science and technology in conformity with graded band gap materials are studied by methods of complex computer simulation. The main goal of the study is analysis and optimization of optical characteristics of infrared photodetectors based on narrow gap semiconductors such as $C d_{x} M n_{y} H g_{1-x-y} T e$.
\end{abstract}

\section{Introduction}

This paper is a brief review of our results in a few directions which are so related that make possible to calculate the nonlinear composition profiles of graded band gap semiconductors with predeterminated optical characteristics. These directions are: phase equilibria in multicomponent systems, Stefan problems of crystal growth, interdiffusion in heterostructures, electron-hole transport in graded band gap semiconductors under illumination and contact phenomena $\left(n^{+}-n\right.$ and $p^{+}-p$ contacts). In the case under consideration the narrow gap photoresistor is studied only.

\section{Phase equilibria in multicomponent systems}

From the practical point of view the first step in the using of concrete semiconductor consists in plotting of phase diagram. Among a number of relatively new semiconductor alloys is of great interest semimagnetic semiconductor $\mathrm{Mn}_{x} H g_{1-x} T e$. The first detailed phase diagram of the ternary $\mathrm{Hg}-\mathrm{Mn}-\mathrm{Te}$ system was plotted [1]. On the base of regular associated solutions theory were calculated solidus lines $(0<x<0.35)$ and liquidus ones for Te-rich corner $0.6<y<0.85$.

\section{Crystal growth of multicomponent systems and Stefan problems}

Crystallization of multicomponent systems in inhomogeneous temperature field leads to inhomogeneous compositional distribution over the thickness of single crystals and epitaxial layers. In narrow gap semiconductors the energy gap depends, to a large extent, on the composition. Thus nonlinear statement of the inverse Stefan problem, 
associated with the determination of the temperature variation law at the boundary in time is very interesting in the connection with construction of solid-state structures posssesing predeterminated characteristics. Special modification of swipping method was developed for numerical solution of such type problems in regions with unknown moving boundary. The computer simulation has made it possible to determine initial compositions of growth solutions, a required degree of their oversaturation and the law of the controlling temperature variation of a growth sell in time for growing epitaxial layers with predeterminated compositional profiles. Crystal growth of $\mathrm{Cd}_{x} H g_{1-x} T e$ under liquid phase epitaxy conditions was considered in [2], $M n_{x} H g_{1-x} T e$ - in [3].

\section{Interdiffusion in heterostructures}

At high temperatures diffusion coefficient of $C d$ in solid is high and close to one in liquid, so under crystal growth conditions interdiffusion changes planned compositional profiles, band parameters and optical characteristics. Hence, determination of interdiffusion coefficient is a nessesary stage in the calculation of real graded structures. For example, it was calculated the nonlinear interdiffusion coefficient for $C d_{x} M n_{y} H g_{1-x-y} T e / C d T e$ in Arrhenius form $D(x, T)=300 \exp (-8.4 x-1.89 / k T)$ $s \mathrm{~m}^{2} / \mathrm{s}$ by special procedure of successive approximations [4]. Boltzmann-Matano procedure [5] is not applicable becouse the thickness of epitaxial layers is very small.

The numerical solution of nonlinear interdiffusion equation in a region with moving boundary is connected with high level of truncation errors. The special modification of iterative implicit swipping method with control of mass integral was developed for these Stefan type problems. This method makes it possible not only to calculate real diffusional profiles under crystal growth conditions but to determine the real time of structure homogenezation on the whole. This homogenezation for vapour phase epitaxy conditions is shown in Fig.1.

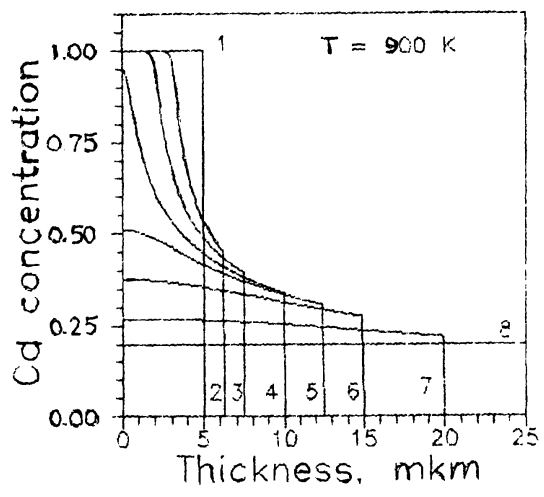

Figure 1. Profiles of $C d$ concentration in growing layer $\mathrm{HgTe} / \mathrm{CdTe}$ in different moments of time

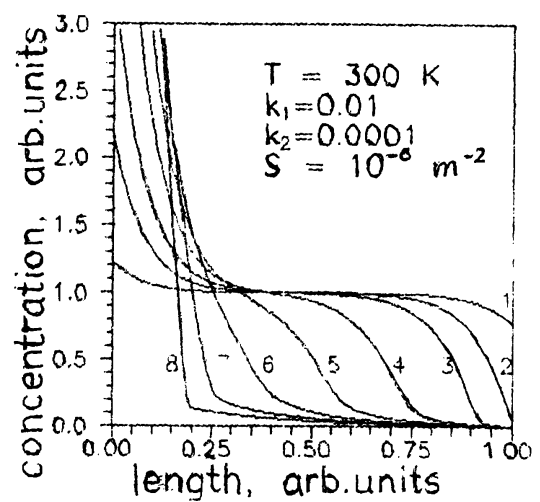

Figure 2. Profiles of electron concentration in the sample for different values of current 


\section{Contact phenomena}

It is necessary to consider phenomena in contacts $n^{+}-n$ or $p^{+}-p$, becouse one does not know any way to use semiconductor structure without outside devices. Following [6], longitudinal exclusion-accumulation model was developed for few special cases and numerical procedure for its investigation was constructed. The idea of obtaining at $300 \mathrm{~K}$ the electron subsystem parameters that may usually be obtained only at liquid nitrogen temperature seems to be very attractive [7]. The typical distributions of carriers over structure thickness are shown in Fig.2. V-A-characteristics were calculated too.

\section{Electron-hole transport in graded band gap semiconductors with nonlinear profile of composition under illumination}

Quasielectric field in graded band gap semiconductors is of great influence on the distribution of nonequilibrium carriers and in fact determines device spectral characteristics. The analysis of the problem shows that construction of devices based on this principle is very perspective. It is often convenient to approximate experimental compositional data by polynoms of high degree and to solve the direct problem of graded structure photoconductivity for determination of final spectral characteristics. But more convenient this approch is for solution of inverce problem. The degree of polynom is determined by number of reper points. By choosing the number and coordinates of reper points one can construct the distribution of nonequilibrium carriers and spectral characteristics needed in special cases $[8,9]$.

Some results of similar computer construction in the case of n-type graded structure under monochromatic light illumination one can see in Fig.3. Fig.3-a shows given types of spectral characteristics, Fig.3-b - compositional profiles needed for arrival at data shown in Fig. 3-a with same curve numbers.

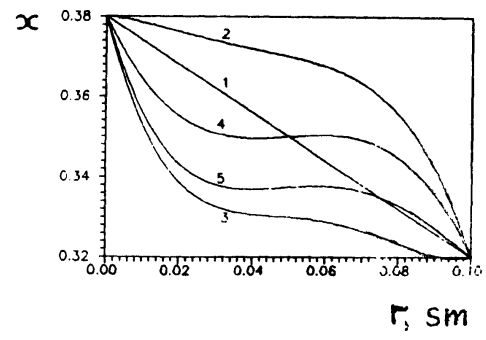

Figure 3-a. Compositional profiles in graded band gap structure $C d_{x} H g_{1-x} T e$

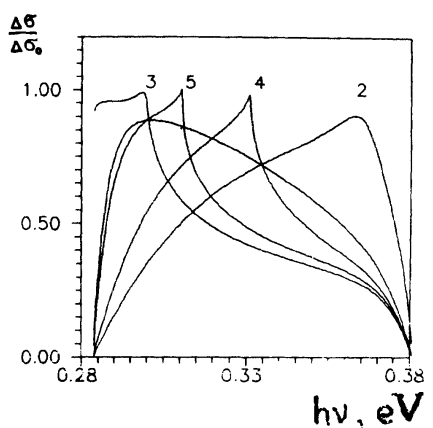

Figure 3-b. Spectral characteristics of these structures with the same numbers of curves on Fig.3-a 


\section{Conclusion}

Of course, it is not any discussion on formulas in this paper. A more concrete statements can be found in references, a description of numerical methods - in more fundamental books, for example in [10]. The most part of our calculations were tested experimentally and by special methods (comparison with exact solutions, conservation of mass integral, steady-state regimes, Runge principle and so on). Theoretical models under consideration are so cumbersome that it is hard to link them mathematically. But particular parts of our simulation can be easily generalized to the more complicated cases for in-depth study and optimization of concrete semiconductor devices. A few program products can demonstrate the approach possibilities in the computer construction of new graded band gap structures.

\section{References}

[1] Zhovnir G.I.,Kletskii S.V.,Sochinskii N.V., Frasunak V.M., - Phase Equilibria in $\mathrm{Mn}-\mathrm{Hg}$-Te System, Izv.AN SSSR, Neorg. mater., 1989, v.25, p.1216-1218 (in Russian).

[2] Zhovnir G.I.,Kletskii S.V., - Numerical Solution of Inverse Stefan Problem, Kristallografia, 1988, v.33, No. 5, p.1271-1273 (in Russian).

[3] Zhovnir G.I.,Kletskii S.V.,Sochinskii N.V., - Computer Simulation of the Inverse Stefan Problem in Conformity with the Liquid Phase Epitaxy of $\mathrm{Mn}_{x} \mathrm{Hg} g_{1-x} \mathrm{Te}$, Phys.stat.sol.(a), 1989, v.115, No. 1, p.K31-K34.

[4] Kletskii S.V.,Sochinskii N.V.,Zhovnir G.I., - Interdiffusion in Heterostructures $C d_{x} M n_{y} H g_{1-x-y} T e / C d T e$, Ukrainian Phys.J. - in press.

[5] Shewmon P.G., - Diffusion in Solids, McGraw-Hill Book Company, N.-Y., 1964.

[6] White A.M., - The Characteristics of Minority-carrier Exclusion in Narrow Direct Gap Semiconductors, Infrared Phys., 1985, v.25, No. 6, pp.729-741.

[7] Ashley T.,Elliot C.T., Harker A.T., - Non-equilibrium Modes of Operation for Infrared Detectors, Infrared Phys., 1986, v.26, No.6, pp.303-315.

[8] Kletskii S.V., - Spectral Characteristics of Graded Band Gap Semiconductors with Nonlinear Profile of Composition, Fizika i tehnika poluprovodnikov, 1992, v.26, No. 9, p.1631-1634 (in Russian).

[9] Kletskii S.V.,Sizov F.F., - $C d_{x} H g_{1-x} T e-G r a d e d$ Band Gap Structures with Nonlinear Profile of Composition, Ukrainian Phys.J. - in press.

[10] Selberherr S., - Analysis and Simulation of Semiconductor Devices, SpringerVerlag, Wien, 1984. 